\title{
Study on Error Identification of New type of Multiple Reading Head Displacement Sensor
}

\author{
WANGxianquan $^{1 \mathrm{a}}$, YUyao $^{2 \mathrm{~b}}$, ZHUguilin $^{3 \mathrm{c}}$, JIANGzenghui $^{4 \mathrm{~d}}$, \\ ZHAOtingting ${ }^{5}$ \\ ${ }^{1}$ Chongqing University of Technology Chongqing College of Electronic Engineering and \\ Automation Chongqing 400054, China . \\ ${ }^{2}$ Chongqing Key Laboratory of Time Grating Sensing and Advanced Testing Technology \\ Chongqing University of Technology Chongqing 400054, China \\ ${ }^{3}$ Chongqing Key Laboratory of Time Grating Sensing and Advanced Testing Technology \\ Chongqing University of Technology Chongqing 400054, China \\ 4. Chongqing Key Laboratory of Time Grating Sensing and Advanced Testing Technology \\ Chongqing University of Technology Chongqing 400054, China \\ a wxq75@qq.com, ${ }^{\mathrm{b}} 1024110332 @ q q . c o m,{ }^{\mathrm{C}} 648422871 @ q q . c o m$
}

\begin{abstract}
Key Word: sensor; multi-measuring head; displacement measurement; error compensation
Abstract: At present Multiple reading head displacement sensor mostly arranged in the form of geometric or uniform method and the accuracy of reading head is very high for fabrication and installation, implementation difficulty. This paper proposed a new method of Multiple reading head measurement under the condition of not increase the difficulty of processing and installation to achieve high precision. Sensor manufacturing, Multiple reading head are arranged in the same stator according to the sensing signal phase arithmetic and sharing a rotor . Allows multiple reading head angular displacement signal and error signal of induction electromotive force with high degree of consistency and shows periodic arithmetic in the phase rule, reduces the difficulty of error identification and correction, improve the measurement precision of the sensor. Experiments shows that: after self-correction with this method, the error of the sensor can be up to \pm 2 "
\end{abstract}

\section{INTRODUCTION}

In the field of displacement sensor detection, Multiple Reading Head often be used in error calibration and compensation to increase the accuracy of sensors. For example, Masuda and Kajitani proposed a method that distributed five reading head in a single index plate to calibrate the error $^{[1]}$. Zhang Shan zhong analyzed theoretically optical dividing head measurement device, when distribute $n$ reading head, it can eliminate all $k=c n$ time error just take the average value as the reading value ${ }^{[2]}$. Dong Feng Su and Xu et al proposed a method to reasonable decorate reading head by analyzing the harmonic of the main grating ruling error and the uniform layout of the reading head, can reduce the main grating ruling error $82.6 \%$ of harmonic component ${ }^{[3]}$. G.X., Zhang et al. used the Grating dividing plate with sine function distribution and install non offset four reading head to correct the error ${ }^{[4] .}$ Masuda and kajitani have six reading head mounted on the main scale in the way of geometric distance.So an angle measurement system based on encoder is developed to realize the error correction of the encoder ${ }^{[5]}$. Zhu Fan and Wu Yiming et al. proposed the method of eliminating the eccentricity of the grating encoder by using the four reading head ${ }^{[6]}$. Wang Wen put forward the installation of 6 reading heads, using the simulated annealing algorithm to eliminate the effect of eccentric angle sensor ${ }^{[7]}$. In the method mentioned above, Multiple Reading Heads will be strictly in accordance with the specific rules of the installation of high precision, installation is more difficult.

In this paper, a new type of grating displacement sensor is proposed, the sensor based on its own structure can form a number of high consistency of the reading head, and the reading head are arranged according to the Arithmetic Phase, So the displacement error on reading head presents periodically arithmetic rules . It is very conducive to the sensor error identification and correction, and improve the measurement accuracy of the sensor. 


\section{SENSOR PRINCIPLE STRUCTURE}

The sensor works in a way of one phase - excited two - phase induction method. Having sinusoidal signal excitation on the excitation winding of the rotor, with the rotation of the rotor, two channels of induction signals which are mutually orthogonal in the stator can be sensed:

$$
\left\{\begin{array}{l}
E_{s}=U \sin \left(\alpha+\beta_{i}\right) \sin (\omega \mathrm{t}) \\
E_{c}=U \cos \left(\alpha+\beta_{i}\right) \sin (\omega \mathrm{t})
\end{array}\right.
$$

In the above expression $E_{S}$ and $E_{c}$ are sinusoidal and cosine winding induced electromotive force, respectively; $\alpha$ is rotor angle with respect to the stator; $\beta_{i}$ is the i groove angle(Electrical angle).

The following have the sensor with $17 \pi / 16$ groove angle in stator as an example to illustrate the method to construct multiple reading heads. Figure 1-1 is the structure of stator slot pitch angle of $17 \pi / 16$ sensor, 16 stator slot, 18 slot rotor.

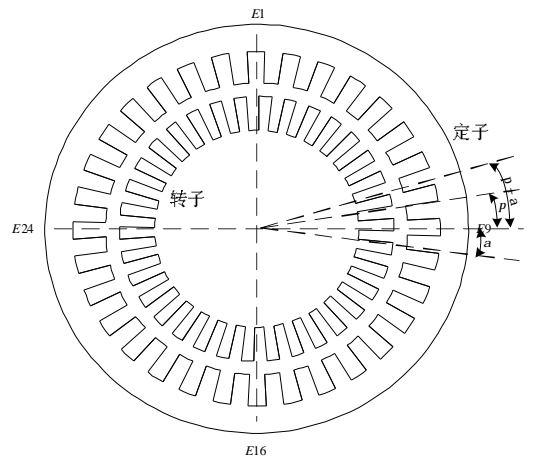

Figure 1-1: Sensor structure

The continuous winding on the rotor is excited by a sinusoidal signal, induced electromotive force in stator slot number $\mathrm{I}$ is:

$$
\mathrm{E}_{\mathrm{i}}=\mathrm{U}_{\mathrm{i}} \cos (\alpha+(\mathrm{i}-1) \times 17 \pi / 16) \sin (\omega \mathrm{t})
$$

In the above expression, $E_{i}$ is induced electromotive force; $U_{i}$-Induced force amplitude; $\alpha$ Electrical angle;

In the Figure 1-1, distributed uniform winding of rotor around the positive and negative, stator winding :

WAY1: $\left\{\begin{array}{l}\mathrm{E}_{1 \mathrm{~S}}=\mathrm{E}_{9}-\mathrm{E}_{25}+\mathrm{E}_{11}-\mathrm{E}_{27} \\ \mathrm{E}_{1 \mathrm{C}}=\mathrm{E}_{1}-\mathrm{E}_{17}+\mathrm{E}_{3}-\mathrm{E}_{19}\end{array} \quad\right.$ (3) WAY2: $\left\{\begin{array}{l}\mathrm{E}_{2 \mathrm{~S}}=\mathrm{E}_{26}-\mathrm{E}_{10}+\mathrm{E}_{28}-\mathrm{E}_{12} \\ \mathrm{E}_{2 \mathrm{C}}=\mathrm{E}_{18}-\mathrm{E}_{2}+\mathrm{E}_{20}-\mathrm{E}_{4}\end{array}\right.$

$E_{i s}, \mathrm{E}_{i c}$-Positive cosine induced electromotive force;

HEAD1:

$$
\left\{\begin{array}{l}
\mathrm{E}_{1 \mathrm{~S}}=-4 \mathrm{U} \sin (\alpha+\pi / 16) \cos (\pi / 16) \sin \omega \mathrm{t} \\
\mathrm{E}_{1 \mathrm{C}}=4 \mathrm{U} \cos (\alpha+\pi / 16) \cos (\pi / 16) \sin \omega \mathrm{t}
\end{array}\right.
$$

HEAD2:

$$
\left\{\begin{array}{l}
\mathrm{E}_{2 \mathrm{~S}}=-4 \sin (\alpha+2 \pi / 8) \cos (\pi / 8) \sin \omega \mathrm{t} \\
\mathrm{E}_{2 \mathrm{C}}=4 \cos (\alpha+2 \pi / 8) \cos (\pi / 8) \sin \omega \mathrm{t}
\end{array}\right.
$$

It can be seen from the output signal: Amplitude of two output signals show the arithmetic rule in phase, the orthogonal signal is constructed on the stator to form a sine and cosine windings, then the output signal of the sine and cosine winding is processed to realize the displacement detection. In the result, the two reading heads just a groove angle displacement difference, the other is exactly the same. This means that a high consistency of the two reading heads is successfully constructed on the stator. This provides a good theoretical basis for the calculation of the angular displacement and the identification and compensation of various errors in the sensor.

After the study found to build sensor method is: for the two-phase sensor and stator slot pitch angle is equal to $p i+p i / 2 n$ ( $n$ is the natural number), for three-phase sensors, stator slot pitch angle is equal to $p i+p i / 3 n$, to appropriate winding way, construct multi head reading the twophase sensor or three-phase sensor. 


\section{SENSOR ERROR IDENTIFICATION}

\section{A. Error analysis of the new sensor}

The new type of sensor contains high harmonic error and low order harmonic error. The highorder errors include the error of function error, amplitude error, quadrature error, two phase output mutual coupling error, coupling error of excitation signal, two phase output phase shift error and so on. Low error caused by the eccentricity and tilt factors such as one, two and three class low error.

\section{(1) Error model of function error}

The following have the sensor with $17 \pi / 16$ groove angle in stator as an example to illustrate the regularity and characteristics of function error. The magnitude of the induced electric potential of the first I slot conductor can be expressed as:

$$
E_{i}=U\left\{\cos \left(\alpha+\beta_{i}\right)+\sum_{\gamma=1}^{N}\left[\mathrm{~A}_{\gamma} \cos \left(\gamma\left(\alpha+\beta_{i}\right)+\mathrm{B}_{\gamma} \sin \left(\gamma\left(\alpha+\beta_{i}\right)\right)\right)\right]\right\}
$$

$\beta_{i}=(\mathrm{i}-1) 17 \pi / 16, \gamma-$ Harmonic frequency; $A_{\gamma}, B_{\gamma}-\gamma$ Harmonic coefficients of harmonic

The reading head 1 , which is composed of a sinusoidal winding and a cosine signal, is a signal (8):

$$
\mathrm{U}_{\mathrm{S}}=\mathrm{E}_{9}-\mathrm{E}_{25}+\mathrm{E}_{11}-\mathrm{E}_{27} \quad \mathrm{U}_{\mathrm{c}}=\mathrm{E}_{1}-\mathrm{E}_{17}+\mathrm{E}_{3}-\mathrm{E}_{19}
$$

The sine and cosine signals are fed into the error amplifier, error function between input angle and output angle such as $(9), \varepsilon_{1}$ is the error caused by function error:

\section{Reading Head 1 is:}

$$
\begin{aligned}
& U_{e 1}=U_{1 S} \cos \left(\alpha_{D}+\pi / 16\right)+\mathrm{U}_{1 c} \sin \left(\alpha_{D}+\pi / 16\right)=U\left[\sin \left(\alpha-\alpha_{D}\right) \cos (\pi / 16)+\varepsilon_{1}\right] \\
& \varepsilon_{1}=\left[\sum_{\gamma=1}^{\mathrm{N}}(-1)^{\frac{\gamma+1}{2}} 4 \mathrm{~A}_{\gamma} \sin \left[\gamma(\alpha+\pi / 16)+(-1)^{\frac{\gamma+1}{2}}(\alpha+\pi / 16)\right]-(-1)^{\frac{\gamma+1}{2}} 4 \mathrm{~B}_{\gamma} \cos \left[\gamma(\alpha+\pi / 16)+(-1)^{\frac{\gamma+1}{2}}(\alpha+\pi / 16)\right]\right] \cos \frac{\gamma}{16} \pi
\end{aligned}
$$

\section{Similarly Reading Head 2 is:}

$$
U_{e 1}=U_{1 S} \cos \left(\alpha_{D}+2 \pi / 16\right)+\mathrm{U}_{1 c} \sin \left(\alpha_{D}+2 \pi / 16\right)=U\left[\sin \left(\alpha-\alpha_{D}\right) \cos (2 \pi / 16)+\varepsilon_{2}\right]
$$

$$
\varepsilon_{2}=\left[\sum_{\gamma=1}^{\mathrm{N}}(-1)^{\frac{\gamma+1}{2}} 4 \mathrm{~A}_{\gamma} \sin \left[\gamma(\alpha+2 \pi / 16)+(-1)^{\frac{\gamma+1}{2}}(\alpha+2 \pi / 16)\right]-(-1)^{\frac{\gamma+1}{2}} 4 \mathrm{~B}_{\gamma} \cos \left[\gamma(\alpha+2 \pi / 16)+(-1)^{\frac{\gamma+1}{2}}(\alpha+2 \pi / 16)\right]\right] \cos \frac{\gamma}{16} \pi
$$

It can be seen from $\varepsilon_{1}$ and $\varepsilon_{2}$ : The error characteristics of the reading head 1 and the first 2 are the same, but the difference is only a little in the phase.

\section{(2) Other high-order error model}

In addition to the error function, the error of amplitude error, the quadrature error, the mutual coupling error of the two phase output, the coupling error of the excitation signal, the phase shift error of the two phase output. The mathematical model of high-order error of two phase induction signal is established, which is the same as the error method of analysis function. Error models are as follow:

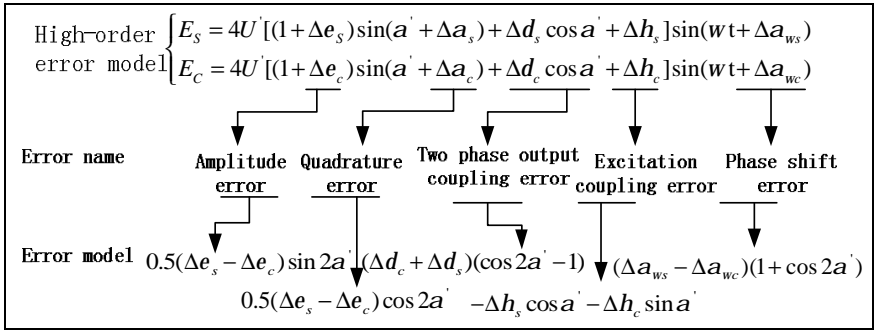

Figure 2-1: High-order error model

Low error caused by the eccentricity and tilt factors such as one, two and three class low error,the error model are as follow: 


$$
\Delta \theta_{i}=\sum_{\gamma=1}^{K}\left(C_{\gamma} \cos \gamma \theta_{i}+D_{\gamma} \sin \gamma \theta_{i}\right)
$$

\section{B Error identification and compensation}

Before identifying and compensating for error, the error must be classified. The error of the new sensor can be divided into two categories:11For Multiple Reading Head, the characteristic of error is consistent;(2)The others error characteristics are different.

For the first class error, We use the two reading heads to establish the error identification model to identify the results, method shows as figure 2-2:

$\left\{\begin{array}{l}\hat{\theta_{1 i}}=\theta_{1 i}-\left(\mathrm{A} \cos \theta_{1 i}+B \sin \theta_{1 i}\right) \\ \hat{\theta_{2 i}}=\theta_{2 i}-\left(\mathrm{A} \cos \theta_{2 i}+B \sin \theta_{2 i}\right) \\ \phi=\hat{\theta_{2 i}}-\hat{\theta_{1 i}} \\ \phi=\hat{\theta_{2 i}}-\hat{\theta_{1 i}}-\left[\left(\operatorname{A} \cos \theta_{1 i}+B \sin \theta_{1 i}\right)+\left(A \cos \theta_{2 i}+B \sin \theta_{2 i}\right)\right]\end{array}\right.$

Figure 2-2 Sketch of error identification

Two identical reading heads $\mathrm{A}, \mathrm{B}$ are separated in an angle. In any position $i$, the result of two heads $\mathrm{A}$ and $\mathrm{B}$ is $\theta_{1 i}, \theta_{2 i}$. Their errors are: $A \cos \theta_{1 i}+B \sin \theta_{1 i}, A \cos \theta_{2 i}+B \sin \theta_{2 i}$. By using "True value $=$ read value- error" establish error identification model, the model have three parameters. Therefore, three or more position information is needed to realize the error parameter identification, which can realize the on-line error correction

If the number of errors in the sensor is increased in the identification model, the number of harmonic coefficients can be increased, and the multiple harmonic error parameters also can be identified.

For the second class error, include direct current component, amplitude error, quadrature error, phase shift error. They are different from the multiple reading heads, which can not be identified. So we use the signal processing method to eliminate the error.

In order to detect the magnitude error, phase shift error and correct the amplitude error, phase shift error and quadrature error, the model of the sensor displacement solver is established. The model of the displacement solver is shown in Figure 2-4.

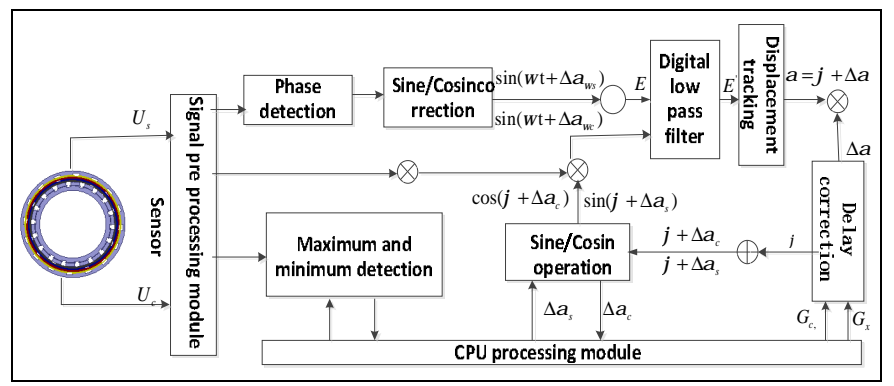

Figure 2-4 Error correction calculation model

\section{SYSTEM SIMULATION AND EXPERIMENTAL RESULT}

\section{A Sensor electromagnetic simulation}

According to the method mentioned above, the sensor model was built in Ansoft software. Sinusoidal excitation of $400 \mathrm{HZ}$ coupled to the excitation winding of the rotor, then set the speed is $15 \mathrm{r} / \mathrm{min}$, observe the change of signal winding voltage signal: 


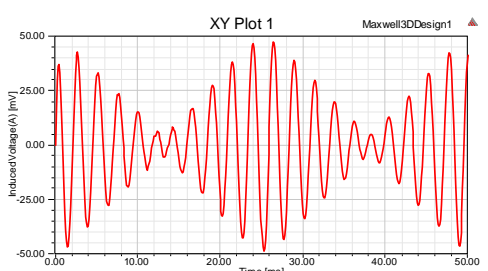

Figure 3-1 Cosine winding output voltage

\section{It can be seen from the figure that the envel} accordance with the sine and cosine variation, and the change of the angular displacement with rotation of the rotor is exactly the case.

\section{B Experimental data acquisition}

The sensor is placed on the high precision precision turntable which is controlled by the servo motor. The dynamic calibration experiment is shown in Figure 3-4.

The turntable for the grating, under the sensor, speed 0.01 RPM / per minute, take the raster data as the true value of the sensor detection point of dynamic testing, and calculate the random error value $^{[8]}$. In the collection, each point of view is collected 36 points, that is, the whole week to collect 12960 points, After the multi reading head error compensation and DSP displacement solution, the error curve is shown \pm 2 in Figure 3-5.

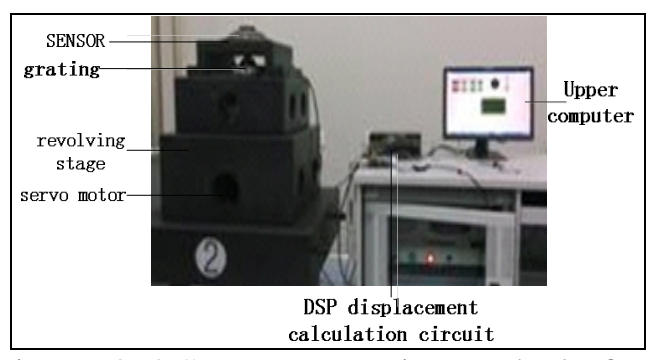

Figure 3-4 Sensor Experimental Platform

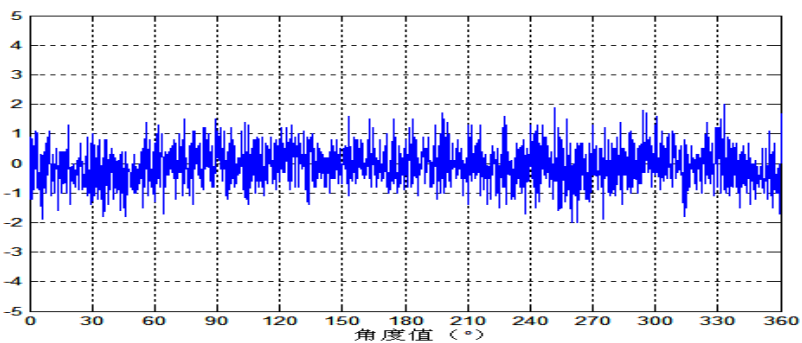

Figure 3-5 Compensated error curve

\section{CONCLUSION}

This design proposes a in does not increase the difficulty of processing and installation of construction of high precision, high consistency of multi reading head displacement sensor and easy to error online self correction of a comprehensive treatment program, to achieve the purpose of high precision angle detection. The scheme is universal, and has wide application value and prospect in the induction of the electromagnetic induction sensor, such as the rotating transformer.

The experimental results show that the tracking error of the final angle can be controlled within \pm 2 "after the online error correction of the multi reading head.

\section{Acknowledgements}

This work was financially supported by the .Project source: National Natural Science Foundation of China (51275551);

\section{REFERENCE}

[1] Watanabe, T., Fujimoto, H., Masuda, T., 2005, Self-Calibration Rotary Encoder, 7th Int., Sym, Meas., Techol. Intellig. Instrum., 240-245.

[2]Zhang Shanzhong. The principle of eliminating the error of multi reading head structure in high precision circular division measurement device [J]. tools and techniques, 1982,06:42-46.

[3] Xu Zhijun; Su Dongfeng et al. To improve the accuracy of the measurement of the circle grating angular measurement system. Journal of electronic measurement and instrument. 2013,27 (7): 653657. 
[4] Zhang, G.X., Wang, C.H., Li, Z. Improving the Accuracy of Angle Measurement System with Optical Grating. Annals of the CIRP. 1994,43(1):457-460.

[5]Masuda,Kajitani. An Automatic Calibration System for Angular Encoders. Precision Engineering. 1989,11(2):95-100

[6] Fanny Chu; Yi Ming Wu; Liu Changchun. By four reading heads eliminate influence of encoder's eccentricity and vibration of Moire fringe phase measurement. Journal of optics.2011,31 (4):04120081-6.

[7] Wang Wen; Lin Keng; Gao Guanbin; Chen Zichen. Eccentricity parameter identification of angle sensor for articulated arm coordinate measuring machine. Optical precision engineering.2010,18: (1): 135-141

[8] Gao Zhonghua, Chen Xihou, Zheng Fangyan. Error correction of time grating angular displacement sensor and its test system [J]. instrument technique and sensor, 2012,09:75-77. 